\title{
ULTRASTRUCTURE OF THE CROWN AND ROOT ODONTOBLASTS
}

M. MoM. Bakri, D.A. Whittaker. Ultrastructure of the crown and root odontoblasts. Annal Dent Univ Malaya 2003; 10: 14-21.

\begin{abstract}
The ultrastructure of the odontoblast reflects the certain phases that the cell undergoes in their lifecycle. Ultrastructure studies of the odontoblasts have often been carried out using young teeth. In this study, teeth from an older individual have been used to study the odontoblasts from the crown and root area. The odontoblasts from the crown area retain their columnar shape while odontoblasts from the root area appeared to be flattened. The organelles present in the odontoblasts either from the crown or root area was observed to be reduced.
\end{abstract}

Key words: ultrastructure, odontoblasts, life-cycle, apical, coronal.

\section{INTRODUCTION}

The morphology of the odontoblast cell in rodent has been described extensively by previous workers $(1,2)$. The odontoblast cell consists of the cell process and cell body. Ultrastructural studies of the cell process have been conducted to determine the extent of the process throughout the entire thickness of dentine $(3,4)$ and it's association with dentine sensitivity $(5,6)$. The ultrastructure of the cell body indicates that they undergo certain phases in their life cycle $(7,8,9)$ and this included the secretory function which has been described by Weinstock and Leblond (2). Couve (10) described the ultrastructural changes in the life cycle of the human odontoblasts as consisting of preodontoblasts, secretory, transitional and aged odontoblast.

Pre-odontoblasts cells are short cylindrical cells (15 p.m) which are developing the intracellular machinery needed for active protein synthesis. In the early pre-odontoblast cell, the intracellular polarity is ill-defined and the nucleus is situated near the basal portion of the cell. The Golgi apparatus is poorly developed, with a few saccules but numerous vesicles are present. Small profiles of rough endoplasmic reticulum can be seen throughout the cytoplasm. Centrioles are located near the Golgi apparatus and form a primary cilium. The function of the cilium is not known but persistence of this feature in the odontoblasts could reflect the static condition of the cell (11) since the primary cilium is common in cells that have completed or withdrawn from the cell-cycle $(12,13)$. In the advanced stages of pre-odontoblasts,

\author{
M. Mohd. BakrP, D.A. Whittaker² \\ IDepartment of Oral Biology, \\ Faculty of Dentistry \\ University of Malaya. \\ 50603 Kuala Lumpur, Malaysia. \\ 2Department of Basic Dental Science \\ Dental School, Heath Park, \\ Cardiff CF4 $4 X Y$, \\ University of Wales College of Medicine \\ United Kingdom. \\ Corresponding author - Marina Mohd. Bakri
}

the Golgi apparatus becomes more prominent and fills much of the supranuclear region. Another normal feature of differentiating odontoblasts is the presence of the cell process that extends from the apical end of the cell (14).

Secretory odontoblasts are organised into a single layer of elongated cells (about 50 p.m in length). They are highly polarised and contain numerous organelles $(2,15)$. Most of the organelles are located mainly in the large supranuclear region with the nucleus at the base of the cell. The large cisternae of the rough endoplasmic reticulum are aligned parallel to the long axis of the cell. The Golgi apparatus comprises of several stacks of saccules. Elongated profiles are the most conspicuous elements of the secretory odontoblasts and these are the presecretory granules. These granules would continue to elongate and become denser to form the secretory granules. Numerous intermediate filaments of about 10p.min diameter are evident in the cyctoplasmic matrix. A system of microfilaments (5-8 '11min diameter) and wide microtubules (27' $11 \mathrm{~m}$ has also been noted by Garant (16).

Transitional odontoblasts are narrower with reduced number of organelles. They are less polarized than in the secretory phase, with the nucleus displaced from the basal extremity and exhibit chromatin condensation. Compared to the secretory odontoblasts, the amount of rough endoplasmic reticulum is reduced and confined to the area around the nucleus. The Golgi apparatus is smaller and located close to the apical region of the nucleus. Autophagic vacuoles are present in the cytoplasm. The autophagic process may be responsible for reorganisation of the cytoplasmic components as it reduces the number of organelles in the cell $(17,18)$.

The last stage in the life cycle of the odontoblasts is represented by the aged odontoblasts. The cells are typically shorter and more crowded than secretory ones, giving the odontoblasts layer a pseudo stratified 
appearance. The organelles of the cell which are reduced in number are mainly located in the infranuclear region. The supra nuclear region is devoid of organelles, except for large lipid-filled vacuoles which are characteristics of the aged odontoblasts. Different procedures of fixation for electron microscopy studies have revealed the presence of large vacuoles, possibly formed from the residual parts of the autophagic process (19). The decrease in size of the rough endoplasmic reticulum and Golgi apparatus, with the absence of secretory granules, suggests that these cells are in the least active stage when compared to the secretory cells.

\section{MATERIALS AND METHODS}

A freshly extracted tooth obtained from a 55 year old person, was sectioned immediately to allow rapid fixation of the tissue. Using the microslicer at low speed with a buffered fixative cooling system, 2 transverse sections from the tooth were obtained; one from the apical region and the other one from the upper third of the root. The fixative used was $2.5 \%$ Glutaraldehyde solution held at a physiological $\mathrm{pH}$ of 7.3-7.4 with the use of a phosphate buffer. The sections were in this fixative at $4^{\circ} \mathrm{C}$ for 3 hours.

The tooth was decalcified using EDTA (Ethylene diamine tetra-acetic acid disodium salt) for approximately three weeks. The end point of the decalcification process was determined by radiographic examination of the specimen. Each of the sections was further reduced to the area surrounding the pulp in order to simplify orientation of the specimen. Tissues were fixed in $2.4 \%$ glutaraldehyde in phosphate buffer at $4 \sim \mathrm{C}$ for 3 hours. This was followed by washing the tissues in 3 changes of buffer over a period of one day. Tissues were then post-fixed in $2 \%$ Osmium
Tetroxide in Veronal Acetate buffer at $4^{\circ} \mathrm{C}$ for 2 hours, dehydrated and embedded in pure resin.

The block containing the pulpal cells to be examined was trimmed with a knife to expose the tissue and sections 0.5-1.5um thick were cut and floated onto a water bath from where they were picked up and mounted onto a labelled glass microscope slide, allowed to dry on a hot plate and stained with $1 \%$ Toluidine blue in $1 \%$ borax. These sections were examined under the light microscope and an appropriate area of the section was selected for further sectioning using the ultramicrotome to produce ultrathin sections. The block face was then retrimmed to include only this area. The sections were gridstained with uranyl acetate and lead citrate before viewing under the Philips CM12 electron microscope.

\section{RESULTS}

\section{Odontoblasts from coronal region}

The odontoblasts, as seen in the toluidine blue stained section, were rather narrow and elongated (Fig 1). Transmission electron microscope examination revealed that the cytoplasm contained parallel cytoplasmic filaments and that profiles of rough endoplasmic reticulum were rarely seen (Figure 4). The mitochondria appeared to be reduced in number and some damage was visible in the mitochondrial cisternae. Tight junctions were visible between adjacent cells.

Cell processes were visible in the odontoblasts tubules (Fig 5) and they were largely devoid of cytoplasmic features except for the presence of filaments and a few small vacuoles. There was a random arrangement of collagen fibrils in the central intertubular zone and fibrils arranged parallel to the cell processes at the periphery of the tubule.

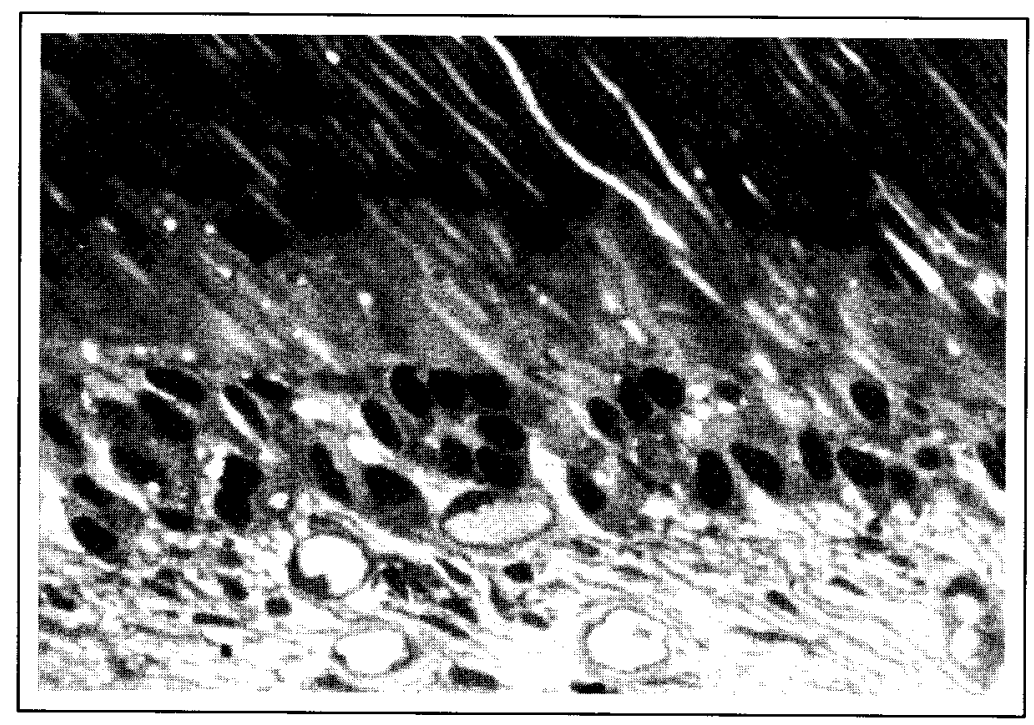

Figure 1: Tolidine blue stained section of the odontoblasts from the coronal area. The shape of the odontoblasts were observed to be rather narrow and elongated. X 40 . 


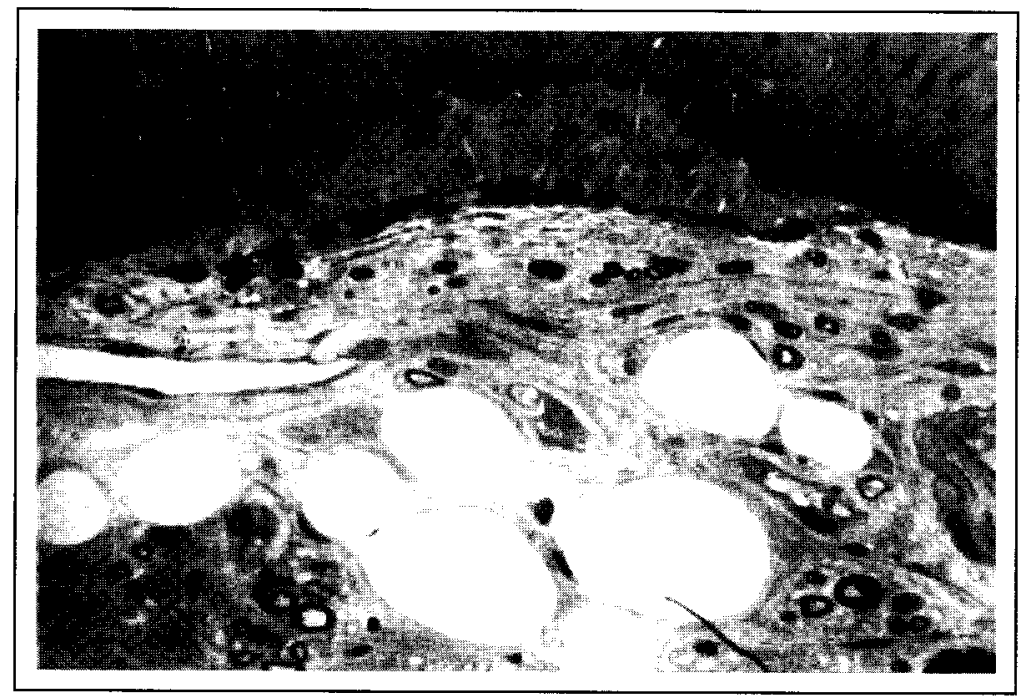

Figure 2: Toluidine blue section of the odontoblasts from the apical area. The odontoblasts were flattened and closely approximated to the pre-dentine zone. X 40 .

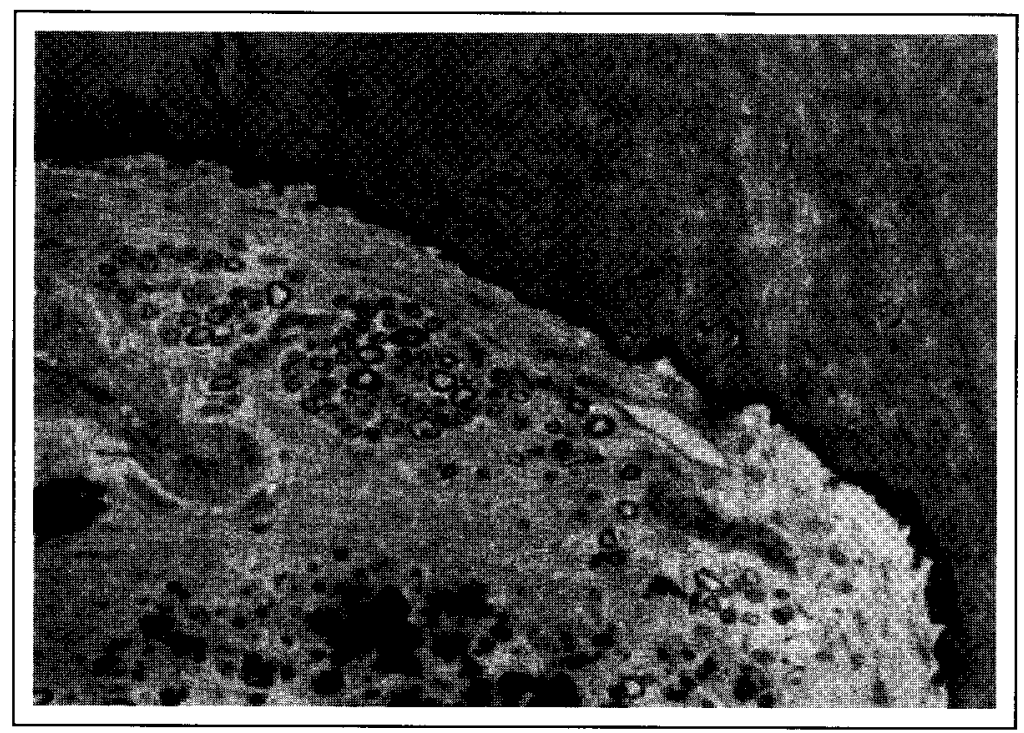

Figure 3: Toluidine blue stained section of the homogenous material observed in the apical area. Where the odontoblasts were not present, the area between the periphery of the pulp and the pre-dentine or the dentine was filled with and homogenous material. X 40.

Odontoblasts from the apical region

In the toluidine blue stained section, it was observed that the cells were few in number and located in specific zones around the pulp periphery (Fig 2). There were some areas between the periphery of the pulp and the dentine or pre-dentine where no odontoblasts can be seen (Fig 3). The odontoblasts were flattened and closely approximated to the predentine zone. Transmission electron microscope examination revealed that tight junctions occurred between adjacent cells and the cytoplasm contained a group of cell organelles in the infra-nuclear region (Fig 6). Large vacuoles were present within the cytoplasm and they contained droplets which, using the present fixation and staining technique may be interpreted as lipid in nature. Only very small amounts of rough endoplasmic reticulum were present within the cytoplasm.

The cell membranes were closely approximated to the pre-dentine and cell processes and could be followed into the pre-dentine zone but not beyond (Fig 6). In the region where odontoblast cell bodies could be readily recognised, both at the light and electron microscopic levels, the pre-dentine consisted of clearly definable collagen fibrils which were densely packed and mainly oriented at right angles to the odontoblastic processes (Fig 7). In zones where no differentiated odontoblast cells were visible, there was a homogenous 


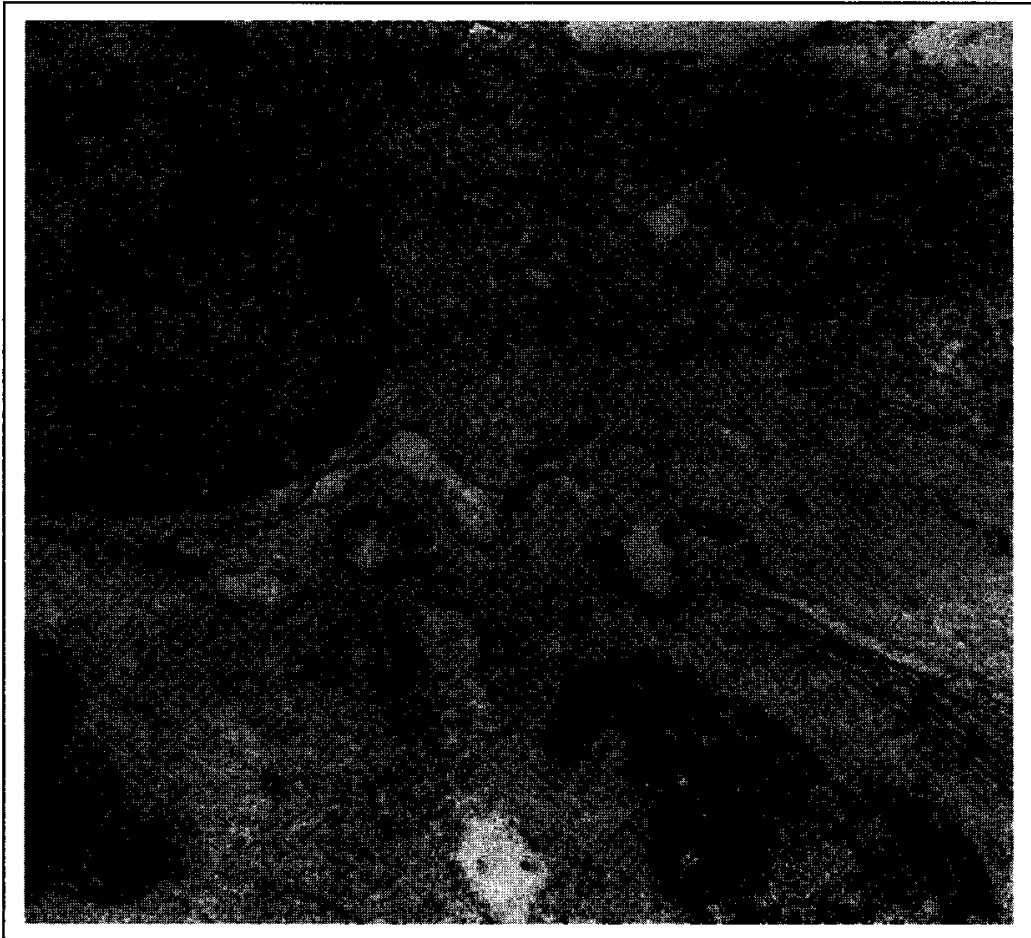

Figure 4: Cell body of the odontoblast from the coronal region. There is reduction in the number of intracytoplasmic organelles. $\mathrm{X} 1700$. Bar $=1.51 \mathrm{~lm}$

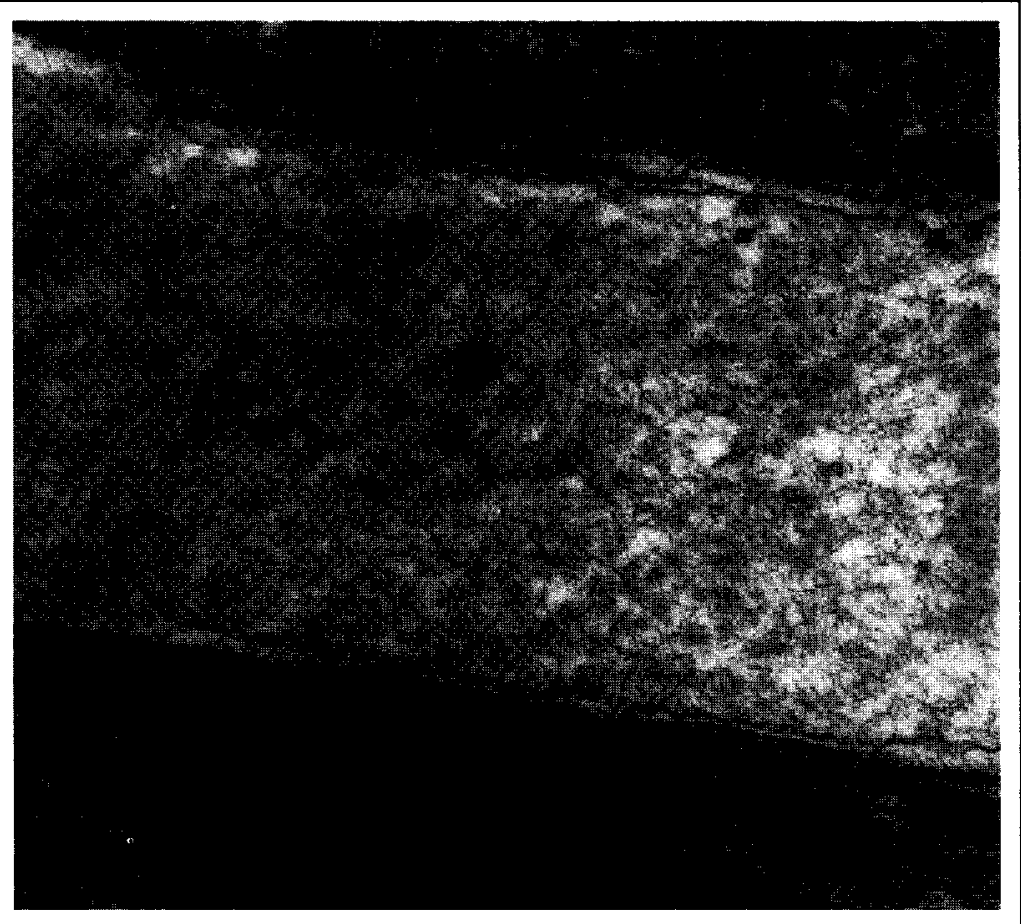

Figure 5: Cell process of the odontoblast from the coronal region. The process was largely devoid of cytoplasmic feature except for the presence of filaments and a few small vacuoles. 


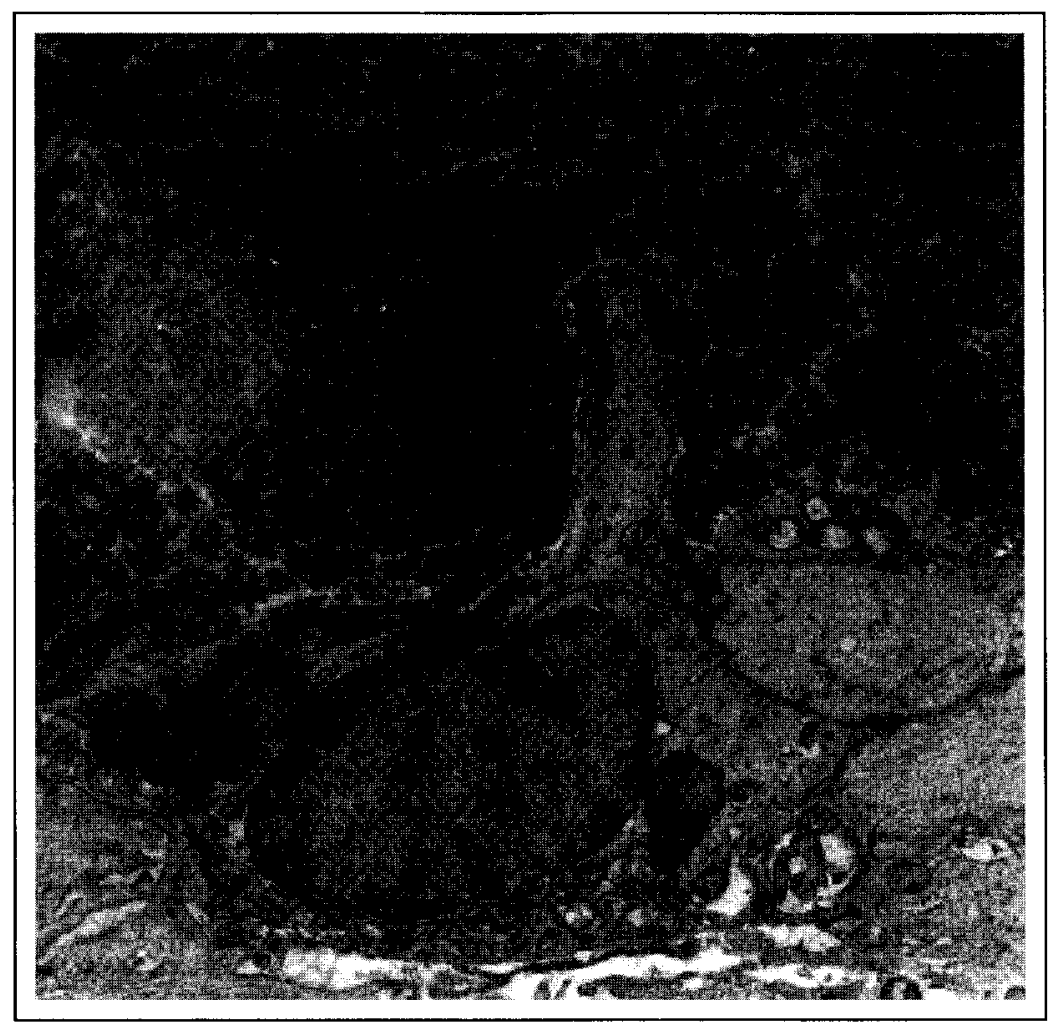

Figure 6: Odontoblast of the apical region. A group of cell organelles can be seen in the infra-nuclear region and very small amounts of rough endoplasmic reticulum were present within the cytoplasm. The cell process could be followed into the pre-dentine zone but not beyond. X 8500. Bar $=311 \mathrm{~m}$.

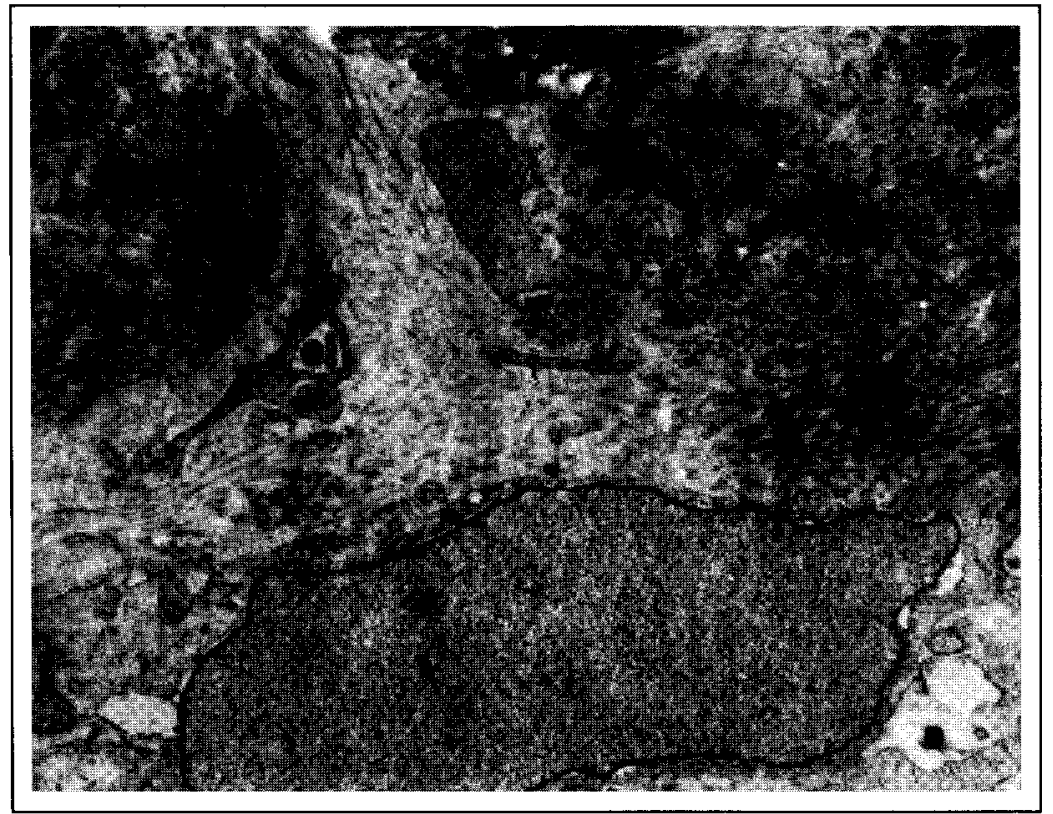

Figure 7: High power view of Fig 6. The pre-dentine consists of densely packed collagen fibrils which were oriented at right angles to the odontoblastic process. $\mathrm{X} 13000$. Bar $=211 \mathrm{~m}$. 


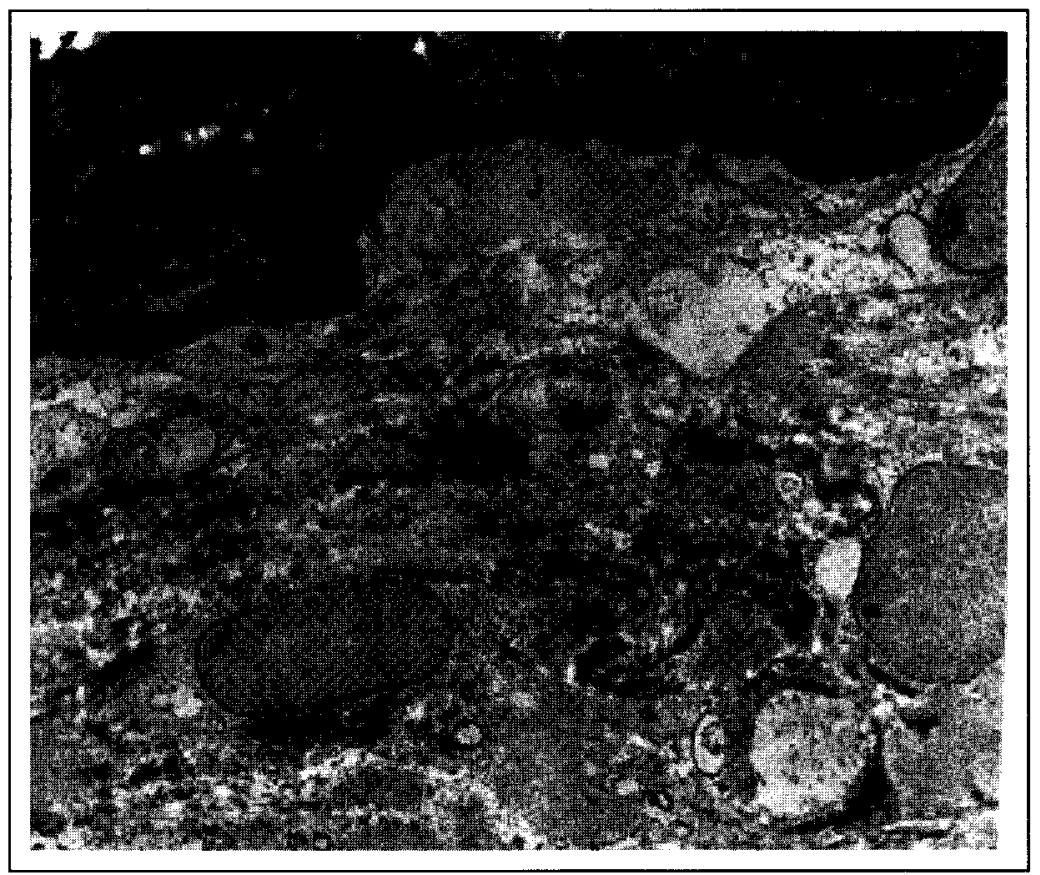

Figure 8: Homogenous material at the border between pulp and predentine.

No differentiated odontoblasts could be seen when this homogenous material was present.

$\mathrm{X}$ 5000. Bar $=51 \mathrm{Lm}$.

zone of material separating the dentine or pre-dentine from the periphery of the pulp (Fig. 8).

\section{DISCUSSION}

Although there appear to have been no previous attempts to study odontoblasts from older individuals, the findings of this study suggest that there are some similarities between the odontoblast of the crown area and the younger coronal odontoblasts described by previous workers. The older odontoblasts from the coronal region in this study retain their columnar shape with reduced number of organelles. The cyctoplasmic reticulum were rarely seen and the number of mitochondria seen was reduced with some damage visible in the mitochondrial cisternae.

In the odontoblast's process, a reduced number of organelles were reported by Harris and Griffin (20) when studying odontoblasts from 25-35 years old patients. They reported the presence of filaments in the odontoblasts process along with a few dilated profiles of rough endoplasmic reticulum and occasional mitochondria. In this study, the number of organelles was noted to be further reduced as apart from the presence of filaments and a few small vacuoles, the odontoblasts processes were largely devoid of cytoplasmic features.

This reduction of organelles seems to occur at an earlier stage of transitional odontoblasts (10) and when compared to the odontoblasts used in this study, is not very marked. De Duve (17) and Glaumann et al (18) proposed that the reduction in the number of organelles seen in the transitional stage as explained by Couve (10) was mediated by an autophagic process where it seems that the process was necessary for reorganisation of the cytoplasmic components. However, Romagnoli et al (21) found that the endoplasmic reticulum undergoes progressive atrophy and the lysosome components decreased in volume in crown odontoblasts from the crown area of rat molars. They, therefore, concluded that autophagocytosis did not occur but instead there was reduction in the renewal of organelles involved in protein synthesis as indicated by the progressive atrophy of the endoplasmic reticulum.

Except for Furseth (19) who studied apical odontoblasts taken from patients 11 to 17 years of age, there appear to have been no attempts to investigate apical odontoblasts from older individuals. In the apical odontoblasts of this present study, the cells were more flattened and closely related to the predentine area. The size and number of cell organelles was reduced and located in the infra-nuclear region. Large vacuoles that were present within the cytoplasm were similar to those described previously $(10,20,22)$ as characteristics of the odontoblasts from the coronal region in the more mature stage of their life-cycle. These vacuoles which sometimes contained lipid droplets might have been the residual part of the autophagic process.

The presence of fat in odontoblasts has been demonstrated histochemically (23) and its presence in the mature odontoblasts may be evidence of maturation or mobilisation of fat arising from trauma. The presence of lipid droplet in vacuoles suggest that in odontoblasts the fat is usually absorbed by a pinocytic 
mechanism and then may be found discharged at the plasma membrane (24), or alternatively, vacuoles containing the droplets may pass out of the cell and disrupt. The appearance of the fat cells may indicate that the cells are in the least active stage of their life cycle.

The outline of the pulp at the apical region was observed to be fairly regular indicating that the deposition of the homogenous material seen at the apical area is keeping pace with the rate of pre-dentine formation by the few odontoblasts left. Although the cellular appearance of these odontoblasts have indicated that they are in the least active stage of their life cycle, they are still productive as shown by the presence of the homogenous material. The homogenous material also contain fibrils further back which implies that there must have been cellular (fibroblast) or odontoblast activity at some stage or else the whole system is coming to a halt and no more pulpal encroachment will occur. As the size of the sample in this study is small, further in depth studies will be required to confirm the findings.

\section{REFERENCES}

1. Garant PR, Szabo G and Nalbandian G. Arch Oral Bioi 1968; 13: 857-76.

2. Weinstock $M$ and Leblond CPo Synthesis, migration and release of precursor collagen by odontoblasts as visualized by autoradiography after 3H-proline administration. J Cell BioI. 1974; 60: 92-127.

3. Holland GR. The extent of the odontoblast process in the cat. J Anat. 1976; 12191: 133-49.

4. Sigal MJ, Aubin IE, Ten cate AR, Pitaru S. the odontoblasts process extends to the dentinoenamel junction: an Immunocytochemical study of rat dentine. J Histochem Cytochem. 1984; 32: 8727 .

5. Holland GR. Morphological features of dentine and pulp related to dentine sensitivity. Arch Oral BioI. 1994; (Suppl 39): 3S-11S.

6. Pashley DH. Dentine permeability and its role in the pathobiology of dentine sensitivity. Arch Oral BioI. 1994; 39 (Suppl): 73S-80S.

7. Murray PE, About I, Lumley PJ, Franquin JC, Windsor LJ and Smith AJ. Odontoblast morphology and dental repair. J Dent 2003; 31: 75-82.
8. Bjorndal L, Darvann $\mathrm{T}$ and Thylstrup A. A quantitative light microscopic study of the odontoblast and subodontoblastic region to active and arrested enamel caries without cavitation. Caries Res 1998; 32: 59-69.

9. Bjorndal L, Darvann T, Bro-Nielsen M, Larsen Rand Thylstrup A. Automated image analysis applied to the odontoblast-predentine region in undemineralized sections of human permanent third molars. Arch Oral Bioi 1997; 42: 329-32.

10. Couve E. Ultrastructural changes during the life cycle of human odontoblasts. Arch Oral Biology. 1986; 31: 643-51.

11. Cameron IL. Cellproliferation and renewal in the mammalian body. In: Cellular and Molecular Renewal in the Mammalian Body 1971. Academic Press, New York. 1971; pp.45-85.

12. Wheatley DN. Cilia in cell-cultured fibroblasts. I. On their occurrence and relative frequencies in primary cultures and established cell lines. J Anat 1969; 105: 351-62.

13. Tucker RW, Pardee AB and Fujiwara K. Centriole ciliation is related to quiescence and DNA synthesis in 3T3 cells. Cell 1983; 17: 527-35.

14. Sisca RF and Provenza DV. Initial dentine formation in human deciduous teeth. Calc. Tiss. Res. 1972; 9: 1-26.

15. Matthiesen ME and Bulow FA von. The ultrastructure of human fetal odontoblasts. Z Zellforsch 1970; 105: 569-78.

16. Garant PRo The organization of microtubules within rat odontoblasts processes revealed by perfusion fixation with glutaraldehyde. Arch Oral Bioi 1972; 17: 1047-58.

17. Duve $\mathrm{C}$ de. The lysosome in retrospect. Lysosomes in biology and pathology; Volume 1. 1969. pp.340. North Holland Publishing, Amsterdam.

18. Glaumann H, Ericsson JLE and Marvella L. Mechanisms of intralysosomal degradation with social reference to autophagocytosis and heterophagocytosis of cell organelles. Int. Rev. CytoI. 1981; 73: 149-82.

19. Furseth R. The fine structure of the odontoblasts / predentine area in the root. Scan J Dent Res 1971; 79: 141-50. 
20. Harris R and Griffin CJ. The fine structure of the mature odontoblasts and cell rich zone of the human dental pulp. Aust Dent J 1969; 14: 16877.

21. Romagnoli P, Mancini F, Galeotti F, Francini E and Pierleoni $\mathrm{P}$. The crown odontoblasts of rat molars from primary dentinogenesis to complete eruption. J Dent Res 1990; 69: 1857-62.

22. Frank RM. Ultrastructural relationship between the odontoblasts, its process and nerve fibres. Dentine and Pulp: their structure and reactions. Livingstone, Edinburgh. 1968; pp.115-45.
23. Symons NBB. Lipid distribution in the developing teeth of the rat. J Dent Res 1957; 36: 805.

24. Franklin DL, Arana-Chavez VE, Katchburian E. Cholesterol in the distal portions of differentiating and fully differentiated rat odontoblasts observed by freeze-fracture. Arch of Oral BioI 1994; 39: 817-9. 